\title{
EMPREGABILIDADE E SATISFAÇÃO DOS DIPLOMADOS DE UMA INSTITUIÇÃO DE ENSINO SUPERIOR: INDICADORES PRELIMINARES
}

\author{
Sónia Brito-Costa \\ Human Potential Development Center, I2A-Institute of Applied Research of Polytechnic Institute of Coimbra \\ sonya.b.costa@gmail.com \\ Rui Antunes \\ Polytechnic Institute of Coimbra \\ antunes@ipc.pt \\ Juan José Maldonado Briegas \\ University of Extremadura \\ juanjose.maldonadob@gmail.com
}

Fecha de Recepción: 11 Septiembre 2018

Fecha de Admisión: 1 Octubre 2018

\section{RESUMO}

0 objetivo deste estudo foi conhecer os índices de empregabilidade e satisfação com a formação numa Instituição de Ensino Superior (IES) numa amostra constituída por 1693 diplomados, com uma média de 29,05 anos de idade (Dp7,67). A análise estatística foi efetuada através do Statistical Package for the Social Sciences (SPSS) versão 20.0 para Windows. Verificou-se que, $72.7 \%$ dos diplomados se encontram empregados, a obtenção desse emprego dá-se na maioria $(82,9 \%)$ até um ao após a conclusão dos estudos e o emprego é maioritariamente (80.9\%) na área de formação, sendo que dos que encontravam empregados à data de conclusão dos seus estudos, a maioria (64.6\%) não mudou ainda de emprego. Quanto à situação contratual atual dos diplomados que se encontram empregados, 55.7\% como trabalhadores por conta de outrem. No que concerne à necessidade de formação complementar à formação académica, $55.1 \%$ do total dos inquiridos referem ter sentido necessidade, mas apenas para progressão na carreira. Quanto à avaliação que os diplomados fazem à sua formação recebida, pela IES no que diz respeito à da adequação da formação obtida no curso que frequentaram para 0 exercício de funções profissionais na área, salienta-se que a maioria (86.3\%) pontuam de "adequada a totalmente adequada". A maioria dos diplomados (92.3\%), considera os conteúdos teóricos e a importância dos mesmos para a prática profissional de "relevante" a "extremamente relevante", 86.1\% quanto à avaliação dos métodos de ensino utilizados, quanto à avaliação da atualização dos programas curriculares pontuam nos $87.4 \%$, e $88.4 \%$ quanto ao estágio curricular. No que concerne à satisfação global com o curso, $81 \%$ dos diplomados inquiridos consideram-se de "satisfeito" a "totalmente satisfeito" relativamente ao curso que frequentaram na IES. 


\section{EMPREGABILIDADE E SATISFAÇÃO DOS DIPLOMADOS DE UMA INSTITUIÇÃO DE ENSINO SUPERIOR: INDICADORES PRELIMINARES}

Palavras Chave: empregabilidade; satisfação com a formação; ensino superior; diplomados

\section{ABSTRACT \\ EMPLOYABILITY AND SATISFACTION OF GRADUATES OF A PORTUGUESE HIGHER EDUCA- TION INSTITUTION: PRELIMINARY INDICATORS}

The aim of this study was to know the indicators of employability and satisfaction with training in a Higher Education Institution (HEI) in a sample of 1693 graduates, with a mean of 29.05 years of age (Dp7,67). Statistical analysis was performed through the Statistical Package for the Social Sciences (SPSS) version 20.0 for Windows. It was verified that, $72.7 \%$ of the graduates are employed, the majority of these graduates $(82.9 \%)$ are employed up to one year after completing their studies, and employment is mostly $(80.9 \%)$ in the training area, being that of those who found employees at the date of completion of their studies, the majority $(64.6 \%)$ did not change jobs yet. Regarding the current contractual situation of the graduates who are employed, $55.7 \%$ as employees. Regarding the need for additional training, $55.1 \%$ of all respondents said they felt a need, but only for career progression. With regard to the evaluation that the graduates make to their training received by the HEI regarding the adequacy of the training obtained in the course they attended to perform professional functions in the area, it should be noted that the majority (86.3\%) score as "adequate" to "fully adequate". The majority of graduates (92.3\%) consider the theoretical contents and their importance for the professional practice of "relevant" to "extremely relevant", $86.1 \%$ regarding the evaluation of the teaching methods used, in evaluating the updating of the programs $87.4 \%$, and $88.4 \%$ on the curricular stage. With regard to overall satisfaction with the course, $81 \%$ of graduates surveyed consider themselves "satisfied" to "fully satisfied" with regard to the course they attended at $\mathrm{HEI}$.

Keywords: employability; satisfaction with training; higher education; graduates

\section{INTRODUÇÃO}

A relação entre a formação que os estudantes recebem numa Instituição de Ensino Superior (IES) e o percurso profissional desses estudantes após a sua graduação é hoje uma variável central na avaliação da qualidade das IES, reconhecida na legislação e nas orientações estratégicas de universidades e politécnicos.

0 Regime Jurídico das Instituições de Ensino Superior (Lei n.. 62/2007, de 10 de setembro)

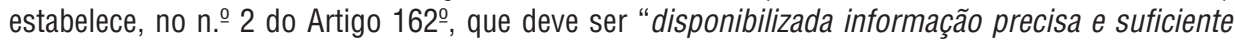
sobre os seguintes aspetos: índices de aproveitamento e de insucesso escolar, bem como de empregabilidade dos ciclos de estudo ministrados".

Por sua vez, o regulamento do Regime Jurídico de Avaliação do Ensino Superior (Lei n.o 38/2007, de 16 de agosto), estabelece na alínea e), ponto ii), do art.-. 18.-. que "as Instituições de Ensino Superior devem publicar, regularmente, informação quantitativa atualizada, imparcial e objetiva acerca da monitorização do projeto dos seus diplomados por um período razoável de tempo, na perspetiva da empregabilidade".

Já a Assembleia da República ao Governo (Resolução da Assembleia da República, n.ํ53/2012 de 23 de abril) indica como "um dos critérios a ser disponibilizado como informação ao candidato do ensino superior a empregabilidade da formação, designadamente: quantos dos formandos por curso estão empregados após a conclusão do curso, quantos se encontram a trabalhar na área de formação, em termos percentuais os que conseguiram emprego através dos serviços de colocação das instituições de ensino".

Também a European University Association ${ }^{1}$ reconhece a importância da empregabilidade quando afirma que "os procedimentos para avaliar a integração e a progressão dos diplomados no mer- 
cado de trabalho após a graduação tornaram-se cada vez mais importantes para as universidades: na idade do ensino superior de massas, ajuda-as a compreender melhor o impacto dos programas de estudos centrados nos estudantes e sua relevância para o mercado de trabalho e, portanto, fornece informações relevantes para a melhoria sistemática de cursos e serviços de suporte."

A avaliação do potencial de empregabilidade e satisfação dos diplomados torna-se assim imperiosa para conhecer o fenómeno. Neste sentido, definiu-se e operacionalização de um modelo para a avaliação anual do potencial de empregabilidade e satisfação dos diplomados de uma Instituição de Ensino Superior (IES).

Foi testado um modelo de recolha num estudo que tem por referência os diplomados de todos os ciclos de estudos ministrados pelas Unidades Orgânicas (UO) da IES no período compreendido entre 2011/2012 a 2015/2016, ou seja, incluindo todos os diplomados de ciclos de estudos e tipo de cursos organizados na sequência do processo de Bolonha.

0 principal objetivo desta primeira fase do estudo é o de definir e testar indicadores de empregabilidade e satisfação com a formação, nomeadamente: (1) 0 tempo decorrido entre a conclusão dos estudos e a obtenção de emprego; (2) a adequação da formação à atividade profissional desempenhada pelos diplomados; (3) o percurso de formação contínua e/ou pós-graduada dos diplomados; e (4) a satisfação dos diplomados relativamente à qualidade e relevância profissional da formação que obtiveram com a frequência do ciclo de estudos.

\section{MÉTODO}

\section{Instrumentos}

0 Questionário utilizado organiza-se em 32 questões e visa obter informação sobre: (1) 0 tempo decorrido entre a conclusão dos estudos e a obtenção de emprego; (2) a adequação da formação à atividade profissional desempenhada pelos diplomados; (3) percurso de formação contínua e/ou pós-graduada dos diplomados; e (4) a perceção dos diplomados relativamente à qualidade e relevância profissional da formação que obtiveram com a frequência do ciclo de estudos..

\section{Recolha de Informação e análise estatística}

A informação foi recolhida através de e-mails enviados a todos os diplomados, utilizando os endereços registados nas bases de Dados da IES. 0 pedido de participação esclarecia os objetivos do estudo, e solicitava a colaboração através do preenchimento de um questionário e forneciam um link para acesso direto ao questionário feito com a aplicação Google Forms e parametrizado de forma a ser preenchido apenas uma vez por cada um dos inquiridos. Depois de um primeiro contacto foram reenviados e-mails a todos os diplomados que não responderam. Deste procedimento foram excluídos os endereços que nos foram devolvidos por serem inexistentes. Procedemos ao reenvio do e-mail por 5 vezes. A análise estatística foi efetuada através do Statistical Package for the Social Sciences (SPSS) versão 20.0 para Windows.

\section{Participantes}

A População que se estudada foram os Diplomados da IES em ciclos de estudos de Licenciatura, Mestrado e em Cursos de Especialização Tecnológica (CET) Cursos de Especialização Pós-Licenciatura e Cursos de Pós-Graduação no período entre 2011/12 e 2015/16. Nestes dados preliminares, que se apresentam apenas podemos usar informação já obtida à data, ou seja, a análise às respostas de 1.693 diplomados da Instituição (Tabela 1).

A amostra ficou constituída por 1693 diplomados, 61.4\% (1040) correspondem ao género feminino e $38.6 \%$ (653) do género masculino. No que concerne às U0, 25.2\% diplomaram-se na U02, $25.2 \%$ na U06, 17.5\% na U03, 16.8\% na U01, $12.6 \%$ na U05 e $2.7 \%$ na Uo4 (Tabela 1). 
EMPREGABILIDADE E SATISFAÇÃO DOS DIPLOMADOS DE UMA INSTITUIÇÃO DE ENSINO SUPERIOR: INDICADORES PRELIMINARES

Tabela 1: Frequência por Unidade de Ensino

\begin{tabular}{rrrrr}
\hline \multirow{3}{*}{ Válido } & \multicolumn{2}{c}{ Frequência } & Percentagem & \% cumulativa \\
\cline { 3 - 5 } & Uo1 & 285 & 16,8 & 16,8 \\
& Uo2 & 427 & 25,2 & 42,1 \\
& Uo5 & 213 & 12,6 & 54,6 \\
& Uo6 & 426 & 25,2 & 79,8 \\
& Uo4 & 45 & 2,7 & 82,5 \\
& Uo3 & 297 & 17,5 & 100,0 \\
& Total & 100,0 & \\
\hline
\end{tabular}

\section{RESULTADOS}

Os indicadores descritivos preliminares agora apresentados referem-se à primeira fase da recoIha de dados e incluem 1.693 respostas obtidas através de contacto por e-mail.

Apresentamos a caracterização de cada indicador relativamente à totalidade dos diplomados respondentes no período de 2011/2012 a 2015/2016.

\section{Distribuição dos Respondentes por Ano Letivo}

No que diz respeito ao ano letivo de conclusão, $15.5 \%$ dos 1693 diplomados que responderam ao questionário terminaram a sua formação no ano letivo de 2011/2012, $14.5 \%$ no ano letivo de 2012/2013, $19.5 \%$ no ano letivo de 2013/2014, $23.5 \%$ no ano letivo de 2014/2015 e $27.0 \%$ no ano letivo de 2015/2016 (Tabela 2).

Tabela 2: Ano de Conclusão

\begin{tabular}{rrrrrr}
\hline \multirow{4}{*}{ Ano } & \multicolumn{1}{c}{ Frequência } & Percentagem & \% válida & \% cumulativa \\
\cline { 3 - 6 } & $2011 / 2012$ & 262 & 15,5 & 15,5 & 15,5 \\
& $2012 / 2013$ & 246 & 14,5 & 14,5 & 30,0 \\
$2013 / 2014$ & 330 & 19,5 & 19,5 & 49,5 \\
$2014 / 2015$ & 398 & 23,5 & 23,5 & 73,0 \\
$2015 / 2016$ & 457 & 27,0 & 27,0 & 100,0 \\
& Total & 1693 & 100,0 & 100,0 & \\
\hline
\end{tabular}

\section{Distribuição dos Respondentes por Ciclo de Estudos}

Quanto ao ciclo de estudos ou grau, verifica-se que 1289 (76.1\%) correspondem a curso de licenciatura, $317(18.7 \%)$ a cursos de mestrado, $48(2.8 \%)$ a CET, $19(1.1 \%)$ a pós-graduação, 19 (1.1\%) a Especializações pós-licenciatura e $1(0.1 \%)$ a CESE (Tabela 3$)$.

Tabela 3: Ciclo/ Grau

\begin{tabular}{lrrrr}
\hline & Frequência & Percentagem & \% válida & \% cumulativa \\
\hline Licenciatura & 1289 & 76,1 & 76,1 & 76,1 \\
Mestrado & 317 & 18,7 & 18,7 & 94,9 \\
CET & 48 & 2,8 & 2,8 & 97,7 \\
Especialização & 19 & 1,1 & 1,1 & 98,8 \\
Pós-Licenciatura & & & 1,1 & 99,9 \\
Pós-Graduação & 19 & 1,1 &, 1 & 100,0 \\
C.E.S.E. & 1 &, 1 & 100,0 & \\
Total & 1693 & 100,0 & & \\
\hline
\end{tabular}




\section{Distribuição dos Respondentes por Situação Profissional}

Quanto à situação profissional, $72.7 \%$ dos diplomados encontram-se empregados, $11.2 \%$ prosseguiram os estudos, 5.4\% encontram-se desempregados à procura do 1ํe emprego, $5.1 \%$ encontram-se desempregados à procura de novo emprego e 5.7\% encontram-se noutra situação (Tabela $4)$.

Tabela 4: Situação profissional atual

\begin{tabular}{lrrr}
\hline & Frequência & Percentagem & \% cumulativa \\
\hline Empregado & 1231 & 72,7 & 72,7 \\
Desempregado, à procura do 1 & 91 & 5,4 & 78,1 \\
emprego & & & 83,2 \\
Desempregado, à procura de novo & 86 & 5,1 & 88,8 \\
emprego & & & 100,0 \\
Outra Situação & 96 & 5,7 & \\
Estudante (Prossegui os estudos) & 189 & 11,2 & 100,0 \\
Total & 1693 &
\end{tabular}

\section{Distribuição dos Respondentes por Compatibilidade entre Formação e Funções}

Dos diplomados respondentes que obtiveram emprego, 80.9\% exercem atualmente funções profissionais compatíveis com o Curso em que se diplomaram, enquanto 19.6\% exercem funções em áreas que não consideram compatíveis com o curso em que se diplomaram (Grafico 1).

Gráfico 1: Exercício de funções profissionais compatíveis com o Curso em que se diplomaram

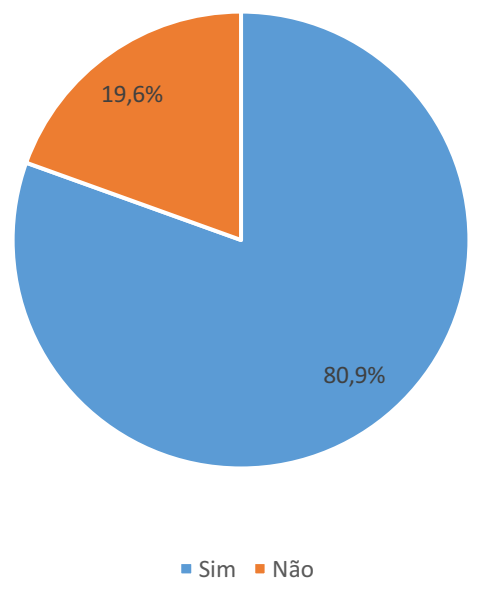

\section{Distribuição dos Respondentes por Tempo para Obtenção de Emprego}

No que diz respeito ao tempo decorrente desde que se diplomaram até à obtenção do seu primeiro emprego remunerado, $82.9 \%$ (percentagem cumulativa) dos diplomados obtiveram o seu emprego até um ano depois de concluírem os seus estudos, sendo de salientar que $29.2 \%$ obtiveram o seu primeiro emprego remunerado em menos de um mês depois da conclusão dos seus estudos, $18.8 \%$ no período de 1 a 3 meses, $18.0 \%$ de 3 a 6 meses e $16.9 \%$ no período de 6 meses a 1 ano depois da conclusão dos seus estudos (Gráfico 2). 


\section{EMPREGABILIDADE E SATISFAÇÃO DOS DIPLOMADOS DE UMA INSTITUIÇÃO DE ENSINO SUPERIOR: INDICADORES PRELIMINARES}

Gráfico 2: Tempo decorrido até ao início do primeiro emprego remunerado como diplomado

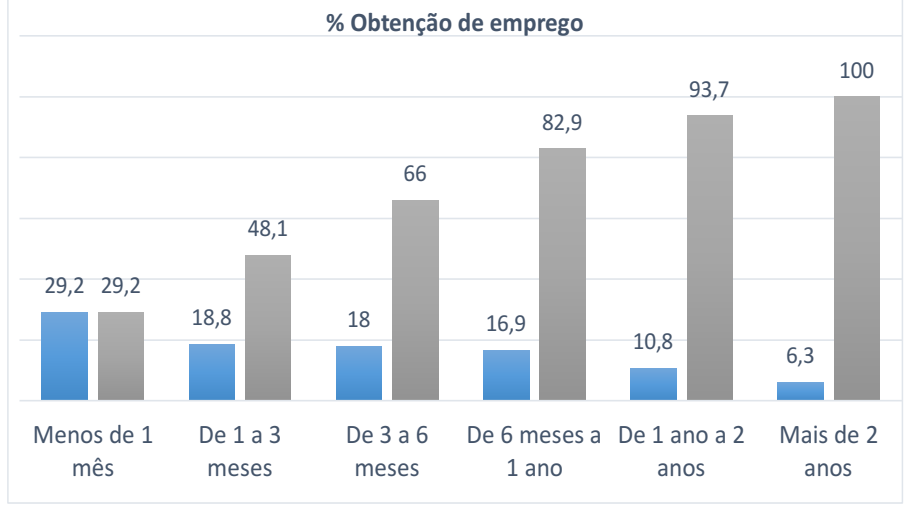

\section{Distribuição dos Respondentes por Oportunidades de Trabalho na Região}

No que concerne às oportunidades de trabalho, 56.6\% (959) dos inquiridos consideram que a região onde vivem oferece oportunidades de trabalho ajustadas ao curso em que se diplomaram, 43.3\% (734) não partilham dessa opinião.

\section{Distribuição dos Respondentes Empregados por Estabilidade no Emprego}

Dos inquiridos que já se encontravam empregados à data de conclusão dos seus estudos, $64.6 \%$ não mudaram ainda de emprego, $16.8 \%$ mudaram de emprego 1 vez, $9.2 \%$ mudaram duas vezes de emprego e $9.4 \%$ já mudaram de emprego 3 ou mais vezes.

\section{Distribuição dos Respondentes Empregados por Situação Contratual}

Quanto à situação contratual atual dos diplomados que se encontram empregados, $7.6 \%$ dos respondentes encontram-se como trabalhadores por conta própria a recibos verdes, $2.5 \%$ como trabalhadores por conta própria (empregador) $55.7 \%$ como trabalhadores por conta de outrem (sector privado), $15.7 \%$ como trabalhadores por conta de outrem (sector público), $1.8 \%$ encontram-se em situação de estágio não remunerado, $11.9 \%$ a realizar estágio remunerado, e 4.9\% encontramse em outra situação.

\section{Distribuiç̧ão dos Respondentes Empregados por Vínculo Contratual}

Quanto ao tipo de vínculo contratual dos diplomados empregados, $48.4 \%$ referem encontrar-se em situação de contrato de trabalho sem termo ou por tempo indeterminado / efetivo, $27.1 \% \mathrm{com}$ contrato individual de trabalho com termo (a prazo), $8.4 \%$ em regime de contrato de prestação de serviços (recibos verdes ou semelhante), $1.9 \%$ encontram-se em situações de trabalho pontuais e ocasionais e $14.2 \%$ encontram-se a realizar estágio

\section{Distribuição dos Respondentes Empregados por Motivação para Procurar Novo Emprego}

Quanto à principal motivação que leva os diplomados atualmente empregados a procurar novo emprego as maiores percentagens de respostas encontram-se nos respondentes que pretendem um emprego mais adequado às suas qualificações académicas $(26.2 \%)$ e nos que pretendem um emprego com remuneração superior (26.1\%), sendo que, 19,7\% dos respondentes referem não querer mudar de emprego e a percentagem mais baixa pertence aos que pretendem um melhor horário de trabalho $(2.3 \%)$. 


\section{Distribuição dos Respondentes por Avaliação da Adequação da Formação}

Quanto à avaliação da adequação da formação obtida no curso que frequentaram para 0 exercício de funções profissionais na área, e numa escala de Likert de 5 pontos que varia entre "totalmente inadequada" e "totalmente adequada", 1.6\% considera a mesma "totalmente inadequada", 12.1\% considera-a "pouco adequada", 48.8\% "adequada", 29.9\% "bastante adequada" e $7.6 \%$ "totalmente adequada", salientando-se que $86.3 \%$ pontuam de "adequada a totalmente adequada" (Gráfico 3).

Gráfico 3: Adequação da formação desenvolvida no Curso, para o exercício da Profissão

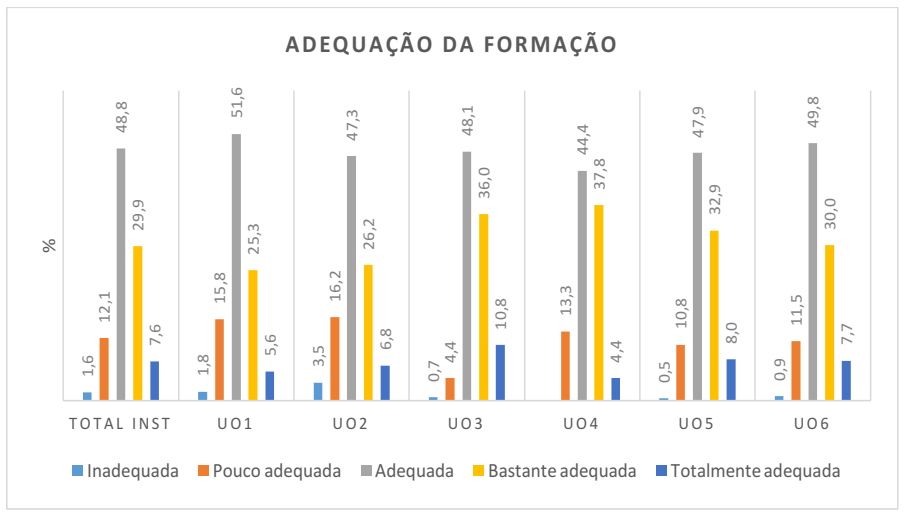

\section{Distribuição dos Respondentes por Necessidade de Formação Complementar}

No que concerne à necessidade de formação complementar à formação académica, $55.1 \%$ do total dos inquiridos referem ter sentido necessidade, $10.8 \%$ não sentiram necessidade, $12.3 \%$ referem ter sentido necessidade, mas apenas para progressão na carreira, enquanto $21.8 \%$ referem que a necessidade que sentiram de recorrer a cursos ou ações de formação se prende com a necessidade de valorização pessoal.

Quanto à frequência de cursos ou ações de formação para complemento da formação académica $55.5 \%$ dos diplomados já frequentaram, sendo que $16.4 \%$ fê-lo para valorização pessoal, e $6.7 \%$ fê-lo com 0 intuito de progressão na carreira.

\section{Distribuição dos Respondentes por Avaliação dos Conteúdos Teóricos do Curso}

No que concerne à avaliação dos conteúdos teóricos do Curso, numa escala de Likert de 5 pontos que varia entre "nada relevantes" a "extremamente relevantes", $6.8 \%$ dos inquiridos pontuam entre "nada relevantes" ( $0.8 \%)$ e "pouco relevantes" $(6 \%)$, e $93.2 \%$ dos inquiridos consideram os mesmos "relevantes"(38.4\%), "bastante relevantes" (43.3\%) e "extremamente relevantes" (11.5\%) (Tabela 5).

Tabela 5: Conteúdos Teóricos do Curso

\begin{tabular}{llrrrr}
\hline & Frequência & Percentagem & \% válida & \% cumulativa \\
\hline Válido & Nada Relevantes & 13 &, 8 &, 8 &, 8 \\
& Pouco Relevantes & 102 & 6,0 & 6,0 & 6,8 \\
Relevantes & 650 & 38,4 & 38,4 & 45,2 \\
Bastante Relevantes & 733 & 43,3 & 43,3 & 88,5 \\
Extremamente Relevantes & 195 & 11,5 & 11,5 & 100,0 \\
Total & 1693 & 100,0 & 100,0 & \\
\hline
\end{tabular}




\section{EMPREGABILIDADE E SATISFAÇÃO DOS DIPLOMADOS DE UMA INSTITUIÇÃO DE ENSINO SUPERIOR: INDICADORES PRELIMINARES}

\section{Distribuição dos Respondentes por Avaliação da Importância dos Conteúdos para a Prática Profissional}

Quanto à importância dos conteúdos para a prática profissional, 7.6\% pontuam de "nada relevantes" a "pouco relevantes", no entanto 92.3\% pontuam de "relevante" a "extremamente relevante” (Gráfico 4).

Gráfico 4: Importância dos conteúdos para a prática profissional

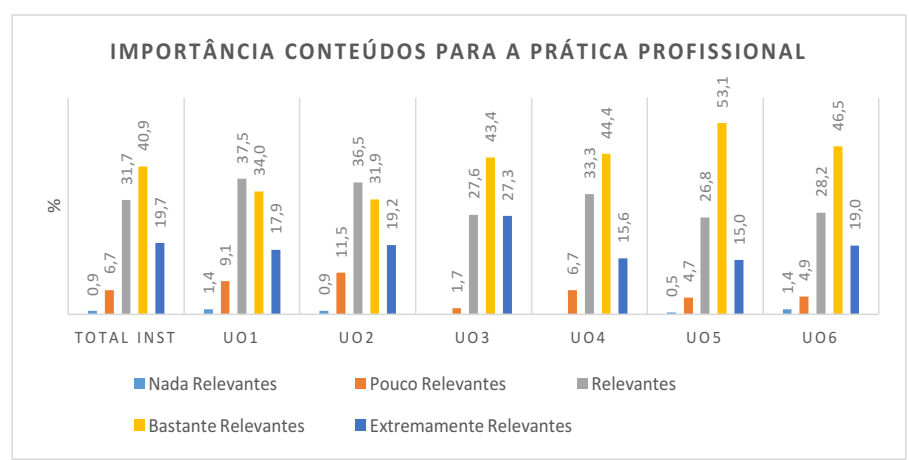

\section{Distribuição dos Respondentes por Avaliação dos Métodos de Ensino}

Quanto à avaliação dos métodos de ensino utilizados, 13.8\% dos inquiridos pontuam de "nada relevantes" (1.4\%) e "pouco relevantes" (12.4\%). No entanto $86.1 \%$ dos inquiridos consideram os mesmos de "relevantes" (40.9\%), "bastante relevantes" (35.9\%) a "extremamente relevantes" (9.3\%) (Gráfico 5).

\section{Gráfico 5: Avaliação dos Métodos de Ensino}

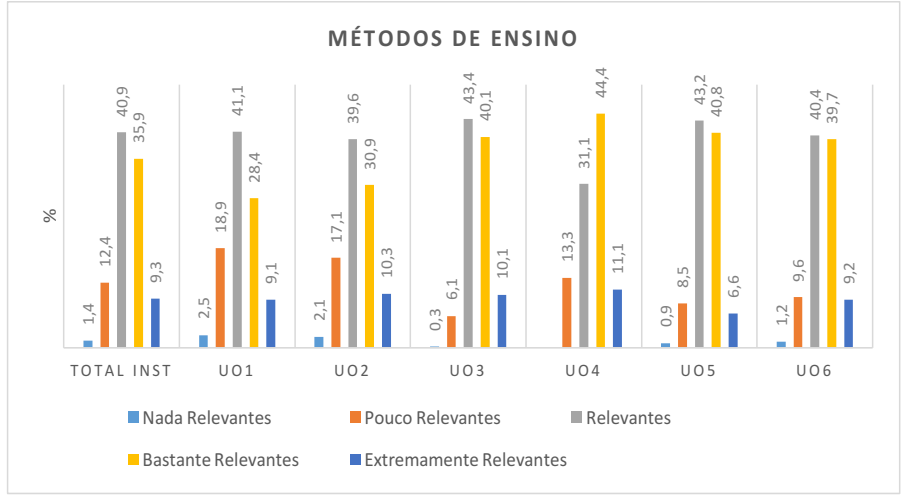

\section{Distribuição dos Respondentes por Avaliação da Importância das Atividades Extracurriculares}

Quanto à realização de atividades práticas extracurriculares, $18.5 \%$ pontuam de "nada relevantes" a "pouco relevantes". No entanto, $81.5 \%$ dos inquiridos pontuam de "relevante" a "extremamente relevante". 


\section{Distribuição dos Respondentes por Avaliação da Atualização dos Programas}

No que diz respeito à avaliação da atualização dos programas curriculares $12.7 \%$ dos inquiridos pontuam "nada relevantes" (2.8\%) e "pouco relevantes" (9.9\%). No entanto $87.4 \%$ dos inquiridos consideram os mesmos de "relevantes" (29.7\%), "bastante relevantes" (34.4\%) a "extremamente relevantes" (23.3\%) (Tabela 6).

Tabela 6: Atualização dos programas curriculares

\begin{tabular}{llrrrr}
\hline & Frequência & Percentagem & \% válida & \% cumulativa \\
\hline Válido & Nada Relevante & 47 & 2,8 & 2,8 & 2,8 \\
& Pouco Relevante & 167 & 9,9 & 9,9 & 12,6 \\
Relevante & 502 & 29,7 & 29,7 & 42,3 \\
Bastante Relevante & 582 & 34,4 & 34,4 & 76,7 \\
Extremamente Relevante & 395 & 23,3 & 23,3 & 100,0 \\
Total & 1693 & 100,0 & 100,0 & \\
\hline
\end{tabular}

\section{Distribuiç̧ão dos Respondentes por Avaliação da Importância do Estágio}

Quanto ao estágio curricular, $11.7 \%$ pontuam de "nada relevante" a "pouco relevante", no entanto $88.4 \%$ dos inquiridos consideram-no de "relevante" a "extremamente relevante" (Tabela 7).

Tabela 7: Estágio curricular

\begin{tabular}{llrrrr}
\hline & Frequência & Percentagem & \% válida & \% cumulativa \\
\hline Válido & Nada Relevante & 111 & 6,6 & 6,6 & 6,6 \\
& Pouco Relevante & 86 & 5,1 & 5,1 & 11,6 \\
Relevante & 272 & 16,1 & 16,1 & 27,7 \\
Bastante Relevante & 422 & 24,9 & 24,9 & 52,6 \\
Extremamente Relevante & 802 & 47,4 & 47,4 & 100,0 \\
Total & 1693 & 100,0 & 100,0 & \\
\hline
\end{tabular}

\section{Distribuição dos Respondentes por Satisfação com o Curso}

No que concerne à satisfação global com 0 curso, $81 \%$ dos diplomados inquiridos consideramse "satisfeitos" (63.3\%) a "totalmente satisfeito" (17.7\%) relativamente ao Curso que frequentaram no IPC (Gráfico 6).

\section{Gráfico 6: Satisfação Global com o Curso}

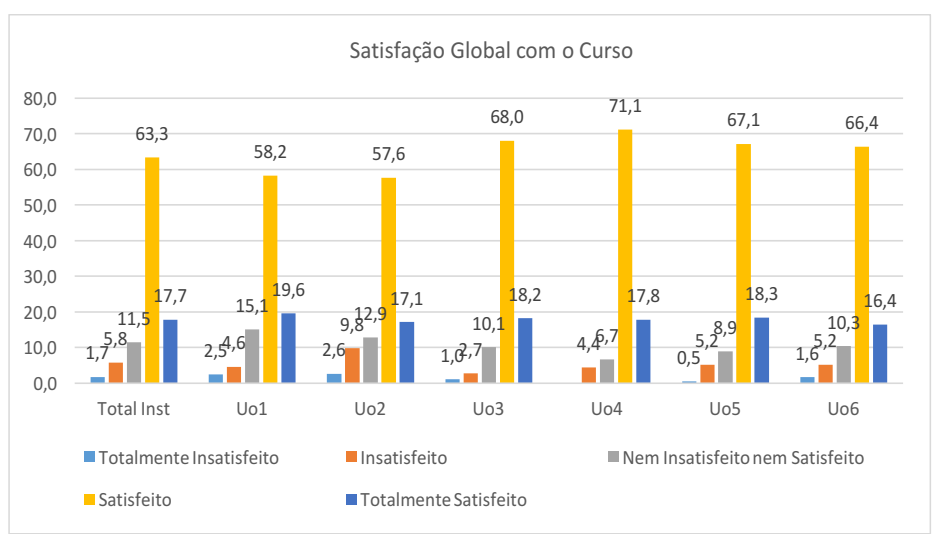




\section{EMPREGABILIDADE E SATISFAÇÃO DOS DIPLOMADOS DE UMA INSTITUIÇÃO DE ENSINO SUPERIOR: INDICADORES PRELIMINARES}

\section{CONCLUSÕES}

0 conhecimento dos percursos e da satisfação dos diplomados é fulcral para qualquer instituição do ensino, dado que permite a compreensão do fenómeno e permite inferir e dinamizar métodos e estratégias potenciadoras do ingresso dos seus diplomados no mercado de trabalho.

A empregabilidade destes diplomados analisou-se de forma transversal e simultânea em todas as Uo. 0 inquérito revelou-se adequado, e permitiu recolher a informação necessária para calcular os indicadores relativos à empregabilidade, no entanto é necessária a constante atualização dos contactos dos diplomados, nomeadamente endereços de e-mail e número de telefone, dado que foi verificado um aumento de respostas à medida que ficou encurtado o tempo entre a data de conclusão do plano de estudos e momento de inquirição, o que demonstra uma maior fidedignidade do registo dos contactos mais recentes, o que facilita, indubitavelmente 0 aumento da taxa de respostas ao inquérito, permitindo inferências e conclusões mais representativas e generalizadas.

A análise incidiu sobre os resultados globais para a IES, não sendo feita a nível de curso por se considerar que a amostra não tinha a dimensão necessária, o que desejamos efetuar futuramente.

Verificou-se que $11.2 \%$ dos diplomados prosseguiram os estudos, e $72.7 \%$ dos diplomados se encontram empregados, sendo o emprego maioritariamente (80.9\%) na área de formação. A maioria dos respondentes (82.9\%) obteve emprego ao fim de 1 ano após terminar a sua formação. Dos inquiridos que já se encontravam empregados à data de conclusão dos seus estudos, a maioria $(64.6 \%)$ não mudou ainda de emprego. Quanto à situação contratual atual dos diplomados que se encontram empregados, $55.7 \%$ como trabalhadores por conta de outrem, $48.4 \%$ referem encontrarse em situação de contrato de trabalho sem termo ou por tempo indeterminado / efetivo, $27.1 \% \mathrm{com}$ contrato individual de trabalho com termo (a prazo).

No que concerne à necessidade de formação complementar à formação académica, $55.1 \%$ do total dos inquiridos referem ter sentido necessidade, mas apenas para progressão na carreira.

Extremamente relevante é a avaliação que os diplomados fazem à sua formação recebida, pela IES. Quanto à avaliação da adequação da formação obtida no curso que frequentaram para o exercício de funções profissionais na área, salienta-se que $86.3 \%$ pontuam de "adequada a totalmente adequada" o que revela uma excelente adequação. No que concerne à avaliação dos conteúdos teóricos do Curso, 93.2\% dos inquiridos consideram os mesmos "relevantes"(38.4\%), "bastante relevantes" (43.3\%) e "extremamente relevantes" (11.5\%). Quanto à importância dos conteúdos para a prática profissional, 92.3\% pontuam de "relevante" a "extremamente relevante". Quanto à avaliação dos métodos de ensino utilizados, $86.1 \%$ dos inquiridos consideram os mesmos de "relevantes" (40.9\%), "bastante relevantes" (35.9\%) a "extremamente relevantes" (9.3\%). Quanto à realização de atividades práticas extracurriculares, $81.5 \%$ dos inquiridos pontuam de "relevante" a "extremamente relevante". No que diz respeito à avaliação da atualização dos programas curriculares $87.4 \%$ dos inquiridos consideram os mesmos de "relevantes" (29.7\%), "bastante relevantes" $34.4 \%$ a "extremamente relevantes" (23.3\%). Quanto ao estágio curricular, $88.4 \%$ dos inquiridos consideram-no de "relevante" a "extremamente relevante". Finalmente, e no que concerne à satisfação global com o curso, $81 \%$ dos diplomados inquiridos consideram-se "satisfeitos" (63.3\%) a "totalmente satisfeito" (17.7\%) relativamente ao Curso que frequentaram na IES.

Estes são, indicadores indubitavelmente positivos para a IES, dado que revelam uma extrema harmonia entre a formação ministrada e as necessidades que os diplomados enfrentam no mercado de trabalho. A obtenção deste tipo de informação fornece à IES um indicador fulcral para a compreensão do fenómeno e identificação de principais lacunas, para que também a IES estimule programas e ações mais adequadas e potenciadoras da inserção profissional dos seus diplomados. 


\section{LEGISLAÇÃO DE INTERESSE E ORIENTAÇ̃̃ES EUROPEIAS}

EUA: European University Association:

EURASHE : European Association of Institution in Higher Education

OFCTESP: Despacho 13057/2016 de 31 de outubro, artº 6 alínea b, c: Acompanhamento diplomados e envolvimento de empregadores

PT 2020 Desafios Educação: Prioridades de Investimento 10.2: Critério 5 Categoria A,4 PO CH candidatura aos CTESP

Resolução da Assembleia da República n.․ 53/2012 de 23 de abril, no 3 alínea b) Critérios acerca da empregabilidade da formação

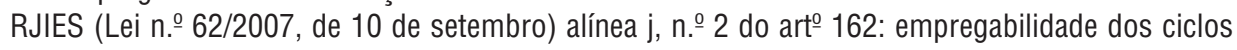
de estudos ministrados

RJAES (Lei n.․ 38/2007, de 16 de agosto), alíneas e, i), ponto ii), do art.- 18. Publicação dados empregabilidade dos diplomados

1 -http://www.eua.be/policy-representation/higher-education-policies/student-tracking-and-employablility 
